\title{
Demonstrating the Role of Anticholinergic Activity in a Mood Disorder
}

\author{
Koji Hori ${ }^{a}$ Kimiko Konishi ${ }^{a}$ b Takahiro Hanashi ${ }^{a}$ Masayuki Tanic Hiroi Tomioka ${ }^{a}$ \\ Yuka Kitajimad $^{d}$ Norihisa Akashi $^{\mathrm{e}}$ Atsuko Inamoto $^{a}$ Kenzo Kurosawa ${ }^{a}$ \\ Sayaka Hasegawa ${ }^{a}$ Takuji Izuno ${ }^{e}$ Nodoka Kikuchi ${ }^{a}$ Misa Hosoi ${ }^{a}$ \\ Mitsugu Hachisu ${ }^{f}$
}

\begin{abstract}
${ }^{a}$ Department of Psychiatry, Showa University Northern Yokohama Hospital, Yokohama, and b Tokyo Metropolitan Tobu Medical Center for Persons with Developmental/Multiple Disabilities, 'Department of Psychiatry, Showa University East Hospital, d Department of Anesthesiology, School of Medicine, Juntendo University, e Department of Psychiatry, Showa University Karasuyama Hospital, and f Department of Pharmaceutical Therapeutics, Division of Clinical Pharmacy, School of Pharmacy, Showa University, Tokyo, Japan
\end{abstract}

\section{Key Words}

Alzheimer's disease - Serum anticholinergic activity . Acetylcholine - Cholinesterase inhibitor - Depression . Mood disorder · Pseudodementia

\begin{abstract}
We report a case of a 54-year-old woman presenting with amnesia, apathy, work-related difficulties and mental stress. At presentation, her Mini-Mental State Examination score was 27 and her serum anticholinergic activity (SAA) was positive without medication or recent physical illnesses. In addition, magnetic resonance imaging revealed mild atrophy of the frontal and temporal lobes, with a relatively intact hippocampus. Consequently, we diagnosed mild cognitive impairment due to Alzheimer's disease and prescribed a cholinesterase inhibitor (donepezil, $10 \mathrm{mg} /$ day); her SAA fully disappeared and clinical symptoms partially resolved. Addition of duloxetine coupled with environmental adjustments caused her cognitive function to return to a normal level, so we diagnosed pseudodementia due to depression. In this case, we believe that the simultaneous cholinergic burden
\end{abstract}

(c) 2015 S. Karger AG, Basel

$1660-2854 / 15 / 0153-0175 \$ 39.50 / 0$ and mental stress led to positive SAA, which made it reasonable to prescribe a cholinesterase inhibitor to ameliorate the associated acetylcholine hypoactivity. We believe that it is essential to recognize the importance of prescribing a cholinesterase inhibitor for specific patients, even those with pseudodementia, to control their clinical symptoms. Moreover, SAA might be a useful biomarker for identifying this subgroup of patients. We propose that anticholinergic activity appears endogenously in mood disorders (depression and bipolar disorder) and set out our rationalization for this hypothesis.

(c) 2015 S. Karger AG, Basel

\section{Introduction}

We have previously proposed the hypothesis of the endogenous appearance of anticholinergic activity (AA) in Alzheimer's disease (AD) [1-3]. To confirm this hypothesis, we need to assess serial long-term serum anticholinergic activity (SAA) [4], a peripheral marker of AA [1-3]. During the process of conducting this re-

\section{KARGER 125}

E-Mail karger@karger.com

www.karger.com/ndd
Koji Hori, MD, PhD

Department of Psychiatry, Showa University Northern Yokohama Hospital 35-1 Chigasakichuo, Tsuzukiku

Yokohama City, Kanagawa 224-8503 (Japan)

E-Mail kojihori@med.showa-u.ac.jp 
search, we encountered an interesting case of a woman initially diagnosed as $\mathrm{AD}$ with mild cognitive impairment (MCI), which was later changed to a diagnosis of pseudodementia due to depression after high-dose cholinesterase inhibitor (ChEI) therapy and antidepressant therapy. Of note, her SAA appeared to be dependent on the treatment and clinical condition, being initially raised before resolving with appropriate therapy. We also discuss why we believe these changes in SAA occurred, together with the rationalization for ChEI therapy, even in pseudodementia, and speculate on the relationship between AA and mood disorder. The patient herself provided written informed consent for the publication of this case report.

\section{Case Presentation}

A 54-year-old woman presented to the memory clinic with a history of memory deterioration. She was a female high school graduate, currently employed as an office worker, with no prior occupational problems. She reported having changed employment 1 year previously, and since then had developed poor relationships with her coworkers, amnesia, apathy and mistakes in her work that had been noted by her employers. Before visiting our clinic, she had been refused treatment by 2 hospitals on the basis that there was no need to treat her memory disturbances. Therefore, she came to our clinic for the treatment of her memory disturbances.

At initial examination, her Mini-Mental State Examination (MMSE) [5] score was 27, and her SAA marker concentration was $6.21 \mathrm{nM}$. Magnetic resonance imaging revealed mild atrophy of the frontal and temporal lobes, with a relatively intact hippocampus. We therefore initially diagnosed MCI [6] on the basis of the criteria developed by the National Institute of Neurological and Communicative Disorders and Stroke in collaboration with the Alzheimer's Disease and Related Disorders Association [7]. We considered her to have early $\mathrm{AD}$ that was likely to progress in the future. Although there was no overt evidence of $\mathrm{AD}$, the memory disturbance, persistent apathy, lack of motivation, mild cognitive atrophy and positive SAA were sufficient to diagnose MCI due to $\mathrm{AD}$, rather than depression.

We prescribed $5 \mathrm{mg}$ /day of donepezil, which significantly and rapidly improved her memory disturbances and apathy. However, because she needed to work, she requested a further dose increase, and following an increase to $10 \mathrm{mg} /$ day, her symptoms almost completely resolved. Approximately 15 months later, her MMSE scores dropped to 25, although her SAA levels were undetectable $(<1.95 \mathrm{nM})$ and she looked outwardly to have returned to the original level. Her MMSE scores and SAA levels remained normal at subsequent follow-ups at 2 and 3 years. During this period, she changed her job and adjusted well to her new work with donepezil as the only treatment. We considered that she had returned to normal levels. However, she said that she felt as if she herself was slightly not complete yet. Therefore, we considered that she was somewhat depressive and we prescribed duloxetine. This treatment led her to a complete resolution of her remaining symptom.

\section{Discussion of This Case}

At her initial presentation, we diagnosed AD for 3 reasons. First, she had several clinical abnormalities that, although nonspecific to $\mathrm{AD}$, are known to be related: subjective memory complaints, and frontal lobe, temporal lobe and hippocampal dysfunction [8,9]. Second, the subjective memory complaints were strong. In fact, despite assessments by our team and another two clinics she persistently refused to accept that her symptoms were not caused by dementia. Nevertheless, some reports have argued that subjective memory complaints are predictive of conversion from normal cognitive function to $\mathrm{MCI}$, and from MCI to $\mathrm{AD}[10]$, so we considered early dementia a possibility. Third, there were objective reports of mistakes at work that had caused her significant employment problems. Together, we initially felt that these facts justified a diagnosis of MCI due to early $\mathrm{AD}$, which was supported by the patient responding to treatment and remaining well. However, during her clinical course, we revised the diagnosis to depression with a pseudodementia.

We previously reported a similar case in which SAA was initially positive in a 74-year-old and became negative 1 year later despite no changes in either medication or physical illness [11]. However, unlike the case presented in this report, her global cognitive function gradually decreased. The addition of antidepressant therapy to the patient in this report facilitated a return to normal cognitive function. We also believe that her global cognitive dysfunction had not returned to normal after 1 year because of residual depression, and that the addition of an antidepressant and adjustment to her work environment led to the complete return of her normal global cognitive function.

AA typically leads to binding of muscarinic acetylcholine $(\mathrm{ACh})$ receptors, causing antagonistic actions on the cholinergic system that lead to deterioration. SAA is a peripheral (tissue) measure of AA [4] that correlates with cerebrospinal fluid levels, thereby reflecting $\mathrm{AA}$ in the central nervous system $[12,13]$. As previously reported, SAA was assessed using the $\left[{ }^{3} \mathrm{H}\right]$ quinuclidinyl benzilate receptor binding assay (Mitsubishi Chemical Medience Corporation, Kumamoto, Japan), following the protocol designed by Tune and Coyle [4]. The origin of SAA was unknown in this patient. Although SAA can be induced by polypharmacy or prescription medications with high AA [14], this patient denied any relevant medication use. Recently, it has been suggested that AA may also appear endogenously due to inflammation [15], mental stress $[16]$ and $\mathrm{AD}[1-3,5]$. 
Fig. 1. We speculate that ACh degradation not only causes cognitive dysfunction and behavioral and psychological symptoms of dementia (BPSD), but also induces inflammation in the central and peripheral nervous systems. This, in turn, induces central and peripheral AA through cytokine pathways. Moreover, AA causes an increase in amyloid levels and further decreases ACh. This is referred to as the 'endogenous AA cascade'. NMDA = N-methyl-D-aspartate. This figure is reproduced from an article by Hori et al. [1]. The procedure was permitted by the Japanese Society of Neuropsychopharmacology (Tokyo, Japan).

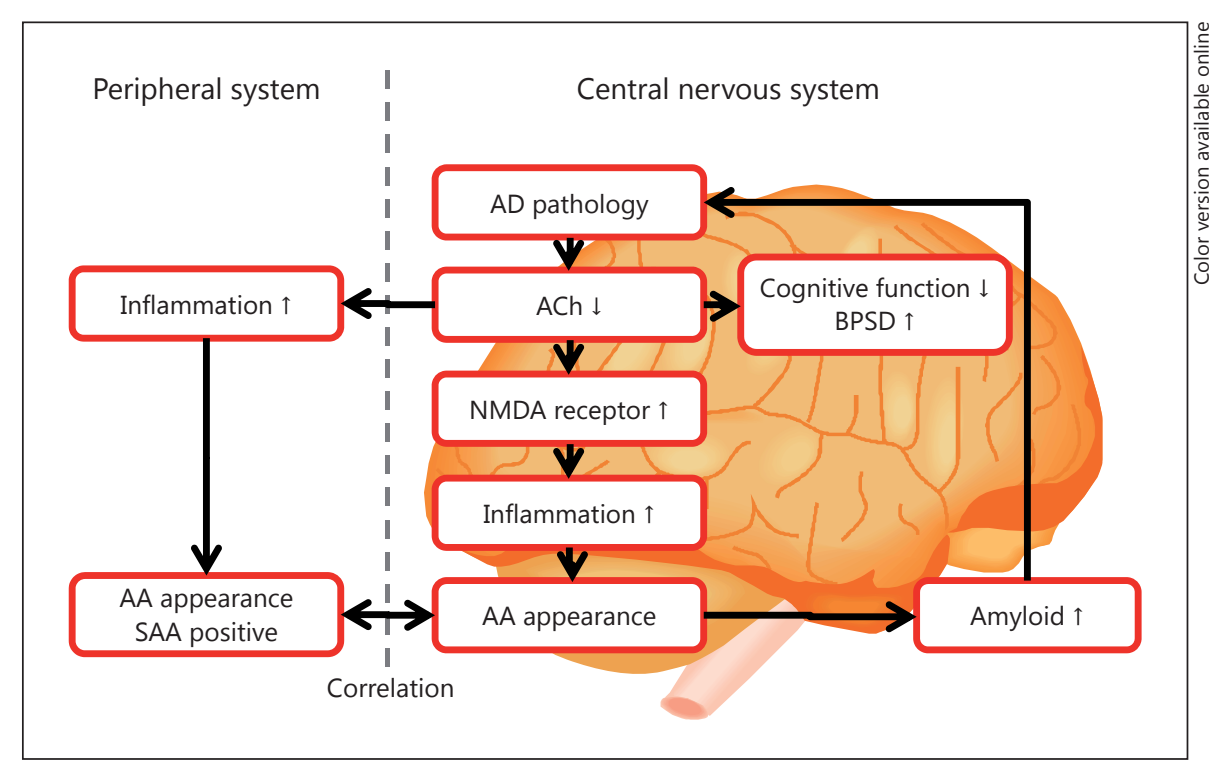

\section{AA in Depression}

We have previously proposed a hypothesis of the endogenous appearance of $\mathrm{AA}$ in $\mathrm{AD}$ [1-3] based on findings that downregulation of $\mathrm{ACh}$ has been implicated in $\mathrm{AD}$ [17] and that ACh regulates the inflammatory system. Indeed, several cytokines resulting from inflammation appear to have AA that accelerates $\mathrm{AD}$ pathology [1] (fig. 1; courtesy of Hori et al. [1]). Therefore, ACh downregulation reaches a critical level in $\mathrm{AD}$, such as in moderate dementia, after which AA appears endogenously. However, given the possibility of AA appearing at an early stage of $\mathrm{AD}[11]$, we speculated that even if the cholinergic system had not deteriorated sufficiently to cause AA (i.e. AD existed insidiously), added stress could prevent cholinergic upregulation, hyperactivity of inflammation and ultimately AA [11]. Our hypothesis was supported by the case report of a 74-year-old woman with a 2-year history of amnesia, who developed visual hallucinations and apathy shortly after moving home. At her first visit to our clinic, her MMSE score was 26 and her SAA marker was $4.28 \mathrm{nM}$, which were ameliorated by $5 \mathrm{mg} /$ day of donepezil for 1 year [11]. We speculate that the appearance of SAA may have been induced in the case of the 74-yearold woman by upregulated inflammatory activity due to mental stress overlapping with preexisting $\mathrm{AD}$ (i.e. $\mathrm{ACh}$ downregulation) [11]. The abolition of AA by ChEI therapy in that case further supported the proposition that AA may be implicated in MCI or early-stage AD [11].

The Role of Anticholinergic Activity in a Mood Disorder
In the present case, the patient reported that she had been an excellent worker before the index episode, and that she was adjusted to her environment. These seemed to preclude a judgment that her cholinergic system was not disordered, but led us to consider why AA had appeared despite an apparently intact cholinergic system. We speculated that her cholinergic system was therefore burdened by some other nonprogressive neuropsychiatric disorder, such as depression, that led to an increase in choline acetyltransferase (ChAT) activity. This supposition was based on a report documenting that ChAT activity could cause a compensatory deterioration in MCI and early $\mathrm{AD}[18,19]$. Indeed, it is possible that this process caused positive SAA results to occur in both the index patient in this report and the patient in the previous report [11]. This patient was probably SAA positive because she experienced both mental stress and burden of the ACh system caused by depression.

These speculations supported our hypotheses that AA could appear endogenously in both AD and mood disorders, with ACh downregulation resulting in SAA. Because the pathology of depression is different from that of $\mathrm{AD}$, there are differences in their symptoms. However, we also speculate that the same mechanisms might work in depression because hyperactivity of ACh causes depressive states [20]. In fact, it has been observed that ACh is related with depressive behaviors [21], while muscarinic receptor bindings were decreased in bipolar disorder and major depressive disorders [22]. We assume that these reports support that in the depressive state, ACh is 

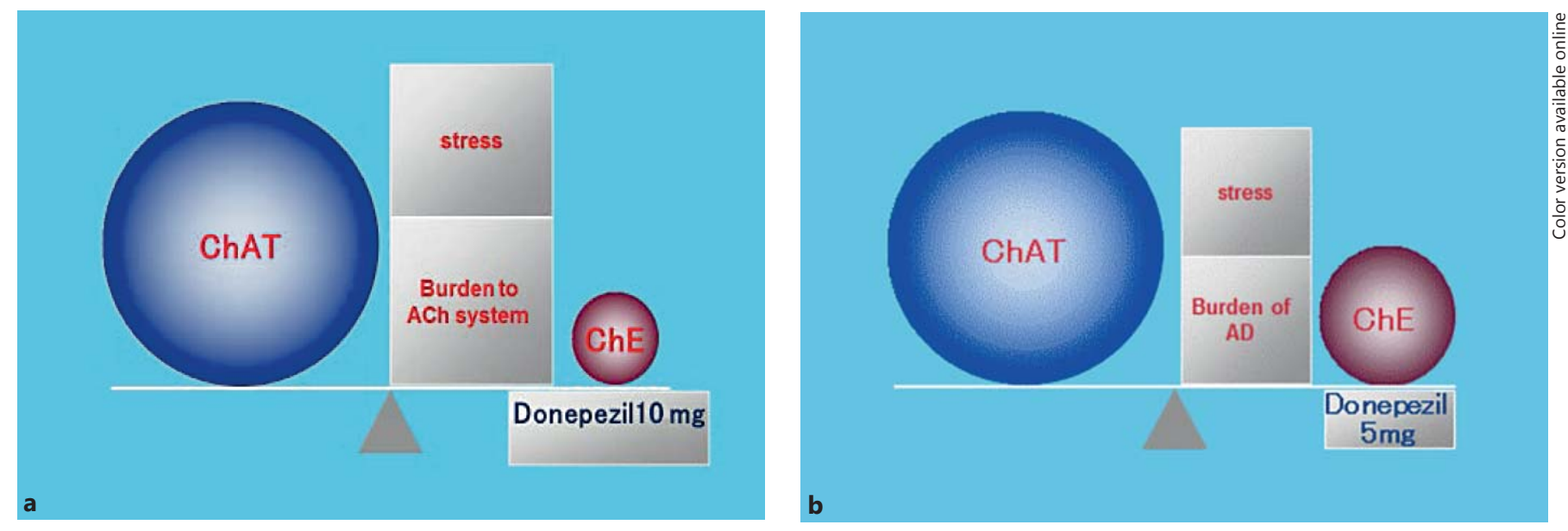

Fig. 2. a A high dose of donepezil ( $10 \mathrm{mg} /$ day) was required to abolish AA by ACh upregulation, even in pseudodementia, because of the strong influence of stress and the burdened ACh system. b Donepezil (5 mg/day) effectively abolished AA by ACh upregulation in a case previously reported by Konishi et al. [11]. ChE = Choline esterase. $\mathbf{a}$ and $\mathbf{b}$ reproduced from Konishi et al. [11], with permission of Karger, Basel, Switzerland.

upregulated and muscarinic receptors are decreased by a compensatory mechanism. So, it is plausible that the ACh system might be burdened or deteriorated in depression, and we speculated that ChEIs could ameliorate the burden to ACh or upregulate $\mathrm{ACh}$ in depression. In the present case, we believe that treatment with a ChEI abolished the AA and ameliorated the associated cognitive dysfunction, while the addition of an antidepressant allowed complete resolution of the remaining clinical symptoms.

Importantly, memory disturbances [23] and apathy [24], which are known to be related to ACh deficiency, can be partially ameliorated with ChEI therapy. In this patient, the SAA marker was $6.21 \mathrm{nM}$, suggesting that she was burdened by AA, regardless of her actual diagnosis. Because her clinical symptoms could be related to ACh deficiency, we considered that it was appropriate to prescribe and continue ChEI therapy, and we increased her dose based on her clinical response. It was noteworthy that ACh upregulation resolved both the clinical symptoms and the SAA. If we recognize this case presentation as valid, we must also recognize that certain patients will require ChEI therapy, especially those with pseudodementia. In some cases, depressive patients are in a pseudodementia state, while in others, the depressive state may relate to MCI (i.e. preclinical dementia).

The anticholinergic burdens in this case and the previous one [11] were 6.21 and $4.28 \mathrm{nM}$, respectively. Therefore, SAA could potentially serve to measure the degree of AA burden in the peripheral and central nervous tissue
$[1,2]$. Moreover, the degree of inflammatory hyperactivity in the present case was approximately 1.5 times higher than that in the patient of the previous study [11]. Therefore, $10 \mathrm{mg} /$ day of donepezil (high dose) was necessary to normalize her SAA through ACh upregulation (fig. 2a), which contrasted with the requirement in the previous report (fig. 2b). In Japan, $5 \mathrm{mg} /$ day of donepezil is prescribed for mild-to-moderate $\mathrm{AD}$, and $10 \mathrm{mg} /$ day is reserved for severe cases only, yet this patient benefited from the higher donepezil dose, despite clinically MCI or early $\mathrm{AD}$. This opinion is supported by observations by Nozawa et al. [25], who reported that $10 \mathrm{mg} /$ day of donepezil was required to relieve symptoms of memory disturbance and apathy related with ACh downregulation.

Our observations lead us to speculate that AA appears endogenously not only in $\mathrm{AD}$, but also in depression and bipolar disorder. In depression, the cholinergic system is burdened secondarily to the underlying depressive pathology. During remission, ChAT activity might be sufficiently upregulated to compensate for the cholinergic burden caused by depressive pathology. Figure 3a summarizes the hypothesis based on the role of 'AA inserts'. In bipolar disorder, we speculate that greater upregulation of ChAT might be needed to compensate for bipolar pathology; figure $3 \mathrm{~b}$ summarizes the endogenous AA hypothesis in bipolar disorder. We do not know whether the difference between depressive and bipolar pathology is qualitative or quantitative. These hypotheses are based on the case report in this article and are only speculative. 
Fig. 3. a In major depression (MD), the cholinergic system is burdened secondarily to depressive pathology. In remission, ChAT activity might be upregulated to compensate for the cholinergic burden caused by depressive pathology. The 'weak AA insert' causes more ChAT hyperactivity, which upregulates ACh to compensate for the AA insert. Upregulation of $\mathrm{ACh}$ then causes depression. However, when the 'strong AA insert' is burdened, ChAT activity is incompletely upregulated in an attempt to compensate for the strong AA insert; in this scenario, ACh is insufficiently upregulated, AA appears, and depression occurs. b In bipolar disorder (BD), we speculate that greater ChAT upregulation might be needed to compensate for bipolar pathology. However, the 'weak AA insert' causes more ChAT hyperactivity, which upregulates $\mathrm{ACh}$ to compensate the AA insert. Upregulation of ACh then causes depression. With the 'strong AA insert', ChAT activity and ACh are not sufficiently upregulated, and AA appears and leads to a manic phase.
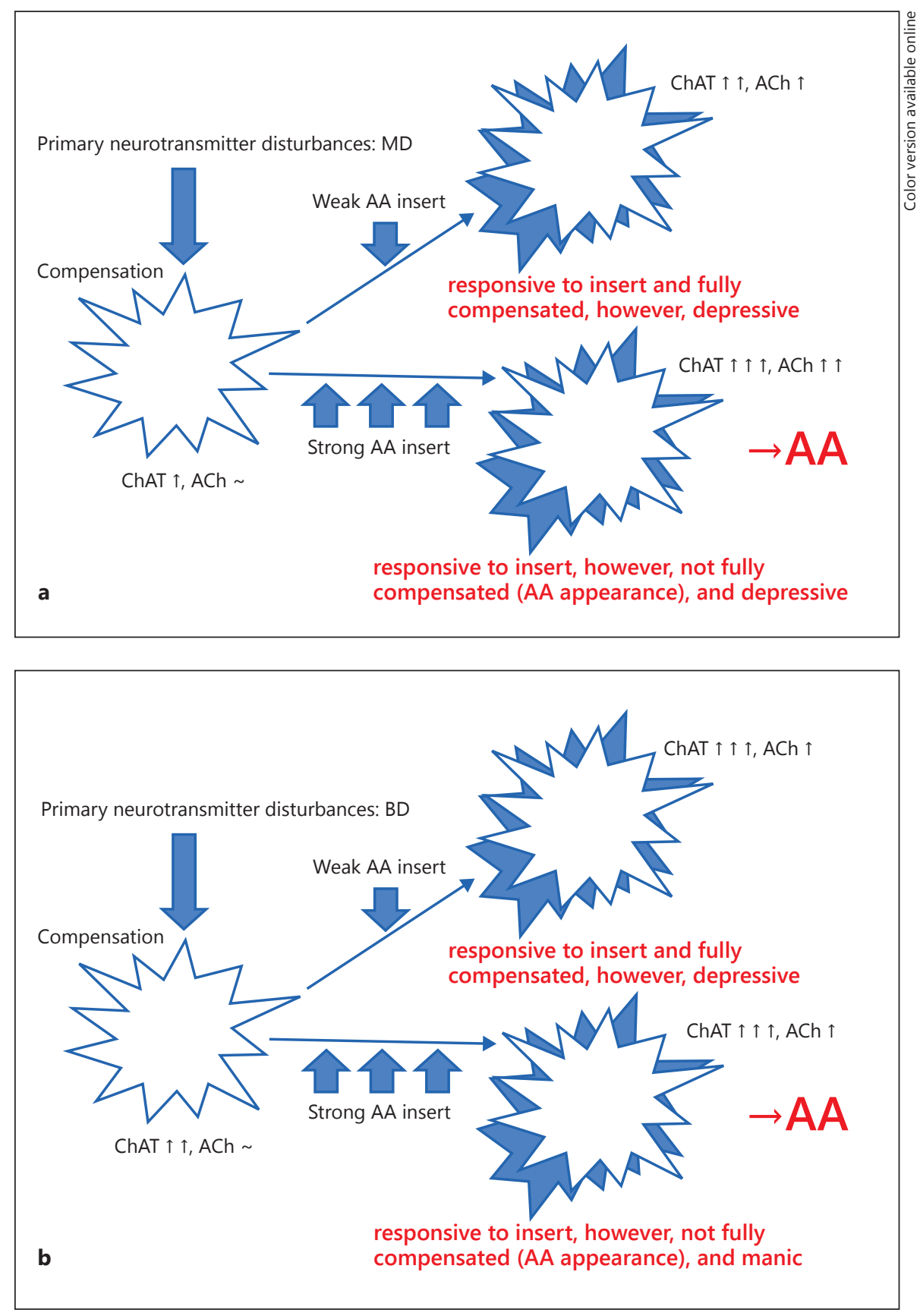

However, we believe that they are compatible with the fact that patients with depression or bipolar disorder tend to suffer from pseudodementia.

We also speculate that autonomic dysfunction is involved in mood disorders, causing deteriorations in the autonomic nervous system, higher hypothalamic-pituitary-adrenal axis activity and, ultimately, hypercortisolism. The plasma cortisol level is high early in the morn-

The Role of Anticholinergic Activity in a Mood Disorder ing and rapidly declines thereafter. We speculate that this rapid decline causes disinhibition of the immune system, with it becoming active in the afternoon, in the evening and at night. If the blood cortisol level rises above normal levels, the subsequent decline can also be expected to be larger, thereby causing a more active inflammatory state and increased AA [2]. Therefore, it is logical that, even if AA does not appear early in the morning, it may appear 
after noon. This mechanism might explain why a patient with typical depression or bipolar disorder has worse depressive symptoms early in the day and relative improvement later in the day. In our model of the relationship between ACh and cortisol, ACh is relatively higher early in the day than later. Consequently, we believe it is biologically plausible that AA appears endogenously in mood disorders.

\section{Conclusion}

Antidementia agents are effective for treating the early stages of $\mathrm{AD}$ and pseudodementia, and some patients need ChEI therapy for amnesia in MCI or pseudodementia. However, as reported by Parnetti et al. [26], caution is required because long-term treatment of antidementia agents to enhance ACh could actually worsen the prognosis in $\mathrm{AD}$. Therefore, it is important to ensure an accurate diagnosis and rationalize therapy. Our results and case experience indicate that SAA may be a good marker for discriminating between those who will and will not benefit from ChEI therapy. There may be a possibility that these hypotheses are overspeculations. However, we constituted these hypotheses step by step. As shown first, we evaluated relationships between AA (or SAA) and clinical symptoms in $\mathrm{AD}$. Based on these results, we speculated on the endogenous hypothesis in AD. Then we reviewed the articles and speculated on the reasons for the endogenous appearance of $\mathrm{AA}$ in $\mathrm{AD}$, i.e. the endogenous $\mathrm{AA}$ cascade in $\mathrm{AD}$. As a third step, in order to demonstrate this endogenous appearance of AA in patients with amnestic symptoms, we observed patients for a long time using SAA. In these processes, we got the opportunity to examine this patient. At her initial presentation, we diagnosed AD. However, during her clinical course, we revised the diagnosis to depression with a pseudodementia.
We considered why AA appeared in this patient with depression, and in this process we speculated on the relationship between AA and depression (mood disorders) reviewing articles including our own. However, this hypothesis is completely dependent on speculations. Therefore, experimentally and basically medical approaches are necessary to identify the substances which have AA and the pharmacokinetics and pharmacodynamics of these substances in order to demonstrate the clinical roles of AA in mood disorders. In addition, a prospective, longterm study with a large sample is needed to confirm or refute our assertions. Moreover, prescribed medications remain an important cause of SAA, so we should also investigate whether SAA can identify those patients on prescribed medications who may or may not benefit from ChEI therapy. It is important to recognize that certain patients appear to benefit from antidementia agents when presenting with amnesia or MCI associated with amnesia, even those with pseudodementia. To this end, SAA might be a useful marker for knowing any patients in need of ChEIs.

\section{Acknowledgments}

Funding for this study was received from Eisai Co. Ltd., Daiichi Sankyo Inc. and Ono Pharmaceutical Co. Ltd.

\section{Disclosure Statement}

Koji Hori received lecture fees from Eisai Co. Ltd., Pfizer Japan Inc., Novartis Pharma KK, Daiichi Sankyo Inc., Ono Pharmaceutical Co. Ltd., Janssen Pharmaceutical KK, Yoshitomi Yakuhin Co. and Mitsubishi Tanabe Pharma Co. Mitsugu Hachisu received funding from Astellas Pharma Inc., Meiji Seika Pharma Co. Ltd., Dainippon Sumitomo Pharm Co. Ltd., Eli Lilly Japan KK and Shionogi \& Co. Ltd., and received lecture fees from Meiji Seika Pharma Co. Ltd. and Mitsubishi Tanabe Pharma Co.

\section{References}

\footnotetext{
1 Hori K, Konishi K, Akita R, Tani M, Tomioka H, Kitajima Y, Yokoyama S, Azuma K, Ikuse D, Akashi N, Yuda H, Hachisu M: Proposal of endogenous anticholinergic hypothesis in Alzheimer's disease (in Japanese). Jpn J Neuropsychopharmacol 2013;33:117-126.

- 2 Hori K, Konishi K, Tani M, Tomioka H, Akita R, Kitajima Y, Aoki M, Yokoyama S, Azuma K, Ikuse D, Akashi N, Hosoi M, Jinbo K, Hachisu M: Serum anticholinergic activity: a possible peripheral marker of the anticholinergic burden in the central nervous system in Alzheimer's disease. Dis Markers 2014;2014: 459013.
}

3 Hori K, Konishi K, Tani M, Tomioka H, Akita R, Kitajima Y, Aoki M, Kikuchi N, Ikuse D, Akashi N, Hosoi M, Jimbo K, Hachisu M: Why does the progression of Alzheimer's disease accelerate? Ann Psychiatry Ment Health 2014:2:1006

4 Tune L, Coyle JT: Serum levels of anticholinergic drugs in treatment of acute extrapyramidal side effects. Arch Gen Psychiatry 1980;37: 293-297.

5 Folstein MF, Folstein SE, McHugh PR: 'Minimental state': a practical method for grading the cognitive state of patients for the clinician. J Psychiatr Res 1975;12:189-198. 
6 Morris JC, Cummings J: Mild cognitive impairment (MCI) represents early-stage Alzheimer's disease. J Alzheimers Dis 2005;7: 235-239.

7 McKhann G, Drachman D, Folstein M, Katzman R, Price D, Stadlan EM: Clinical diagnosis of Alzheimer's disease: report of the NINCDS-ADRDA Work Group under the auspices of Department of Health and $\mathrm{Hu}$ man Services Task Force on Alzheimer's Disease. Neurology 1984;34:939-944.

-8 Stewart R, Dufouil C, Godin O, Ritchie K, Maillard P, Delcroix N, Crivello F, Mazoyer B, Tzourio C: Neuroimaging correlates of subjective memory deficits in a community population. Neurology 2008;70:1601-1607.

$\checkmark 9$ Eskildsen SF, Coupé P, Fonov VS, Pruessner JC, Collins DL; Alzheimer's Disease Neuroimaging Initiative: Structural imaging biomarkers of Alzheimer's disease: predicting disease progression. Neurobiol Aging 2015; 36(suppl 1):S23-S31.

10 Gifford KA, Liu D, Lu Z, Tripodis Y, Cantwell NG, Palmisano J, Kowall N, Jefferson AL: The source of cognitive complaints predicts diagnostic conversion differentially among nondemented older adults. Alzheimers Dement 2014;10:319-327.

-11 Konishi K, Hori K, Tomioka H, Minegishi G, Tani M, Tanaka H, Akita R, Yokoyama S, Oshio T, Hachisu M: Donepezil abolishes anticholinergic activity in a patient with amnesia. Pharmacology 2013;91:86-91.

12 Miller PS, Richardson JS, Jyu CA, Lemay JS, Hiscock M, Keegan DL: Association of low serum anticholinergic levels and cognitive impairment in elderly presurgical patients. Am J Psychiatry 1998;145:342-345.
13 Plaschke K, Thomas C, Engelhardt R, Teschendorf $\mathrm{P}$, Hestermann U, Weigand MA, Martin E, Kopitz J: Significant correlation between plasma and CSF anticholinergic activity in presurgical patients. Neurosci Lett 2007; 417:16-20.

14 Tune L, Carr S, Hoag E, Cooper T: Anticholinergic effects of drugs commonly prescribed for the elderly: potential means for assessing risk of delirium. Am J Psychiatry 1992;149: 1393-1394.

15 Flacker JM, Lipsitz LA: Serum anticholinergic activity changes with acute illness in elderly medical patients. J Gerontol A Biol Sci Med Sci 1999;54:M12-M16.

16 Plaschke K, Kopitz J, Mattern J, Martin E, Teschendorf P: Increased cortisol levels and anticholinergic activity in cognitively unimpaired patients. J Neuropsychiatry Clin Neurosci 2010;22:433-441.

17 Whitehouse PJ, Price DL, Struble RG, Clark AW, Coyle JT, Delon MR: Alzheimer's disease and senile dementia: loss of neurons in the basal forebrain. Science 1982;215:12371239.

18 Gilmor ML, Erickson JD, Varoqui H, Hersh LB, Bennett DA, Cochran EJ, Mufson EJ, Levey AI: Preservation of nucleus basalis neurons containing choline acetyltransferase and the vesicular acetylcholine transporter in the elderly with mild cognitive impairment and early Alzheimer's disease. J Comp Neurol 1999;411:693-704.

19 De Kosky ST, Ikonomovic MD, Styren SD, Beckett L, Wisniewski S, Bennett DA, Cochran EJ, Kordower JH, Mufson EJ: Upregulation of choline acetyltransferase activity in hippocampus and frontal cortex of elderly subjects with mild cognitive impairment. Ann Neurol 2002;51:145-155.
20 Jaffe RJ, Novakovic V, Peselow ED: Scopolamine as an antidepressant: a systematic review. Clin Neuropharmacol 2013;36:24-26.

21 Mineur YS, Obayemi A, Wigestrand MB, Fote GM, Calarco CA, Li AM, Picciotto MR: Cholinergic signaling in the hippocampus regulates social stress resilience and anxiety- and depression-like behavior. Proc Natl Acad Sci USA 2013;110:3573-3578.

22 Gibbons AS, Scarr E, McLean C, Sundram S, Dean B: Decreased muscarinic receptor binding in the frontal cortex of bipolar disorder and major depressive disorder subjects. J Affect Dis 2009;116:184-191.

23 Rokem A, Silver MA: The benefits of cholinergic enhancement during perceptual learning are long-lasting. Front Comput Neurosci 2013;7:66.

24 Ishizaki J, Mimura M: Dysthymia and apathy: diagnosis and treatment. Depress Res Treat 2011;2011:893905.

25 Nozawa M, Ichimiya Y, Nozawa E, Utumi Y, Sugiyama H, Murayama N, Iseki E, Arai H: Clinical effects of high oral dose of donepezil for patients with Alzheimer's disease in Japan. Psychogeriatrics 2009;9:50-55.

26 Parnetti L, Chiasserini D, Andreasson U, Ohlson M, Hüls C, Zetterberg H, Minthon L, Wallin AK, Andreasen N, Talesa VN, Blennow K: Changes in CSF acetyl- and butyrylcholinesterase activity after long-term treatment with AChE inhibitors in Alzheimer's disease. Acta Neurol Scand 2011;124:122129. 